\title{
Comparing MMPI-2-Restructured Form Scores by Service Era for Veterans Assessed Within the Veteran Affairs Healthcare System
}

\author{
Paul B. Ingram ${ }^{1,4}$ (D) Anthony M. Tarescavage ${ }^{2} \cdot$ Yossef S. Ben-Porath ${ }^{3} \cdot$ Mary E. Oehlert $^{4}$
}

Published online: 30 August 2019

(c) Springer Science+Business Media, LLC, part of Springer Nature 2019

\begin{abstract}
This study compares profiles of Minnesota Multiphasic Personality Inventory-2-Restructured Form (MMPI-2-RF) scale scores from 1492 VA test-takers who served during the Vietnam or Gulf War service eras. The sample includes all such cases collected at any VA posttraumatic stress disorder Clinical Teams across the United States between January 1, 2008 and May 31, 2015 using the MMPI-2 or MMPI-2-RF (via the VA Mental Health Assistant suite). Associations between gender and score differences were also examined. In contrast to past research using the MMPI-2, results of this study suggest that veterans are generally homogeneous in their MMPI-2-RF profiles across different periods of service. Specifically, the magnitudes of mean differences are small and not clinically significant. Thus, responses on the MMPI-2-RF do not appear influenced by service era. Implications for the clinical use of, and research with, the MMPI-2-RF are discussed within the VA healthcare system.
\end{abstract}

Keywords MMPI-2-RF · Veteran Affairs $\cdot$ Psychological assessment $\cdot$ Service era

The Veteran Health Administration (VHA) provides medical and psychological care for a large portion of the country's veterans. There are approximately 18.5 million veterans presently in the United States and about $21 \%$ have mental or physical health conditions resulting from, or exasperated by, their military service (United States Census Bureau, 2017). Accordingly, those who are service connected frequently receive compensation for multiple conditions, including both physical and mental health diagnoses (Veterans Benefit Administration, 2017) and a variety of services options exist

The views expressed in this manuscript do not reflect those of the Department of Veteran Affairs or of the United States Government.

Paul B. Ingram

pbingram@gmail.com

1 Department of Psychological Sciences, Texas Tech University, Lubbock, TX, USA

2 Department of Psychology, John Carroll University, University Heights, OH, USA

3 Department of Psychological Sciences, Kent State University, Kent, OH, USA

4 Dwight D. Eisenhower VAMC, Eastern Kansas Veteran Healthcare System, Leavenworth, KS, USA within the Veteran Affairs (VA) system to address veteran healthcare concerns (e.g., routine and emergency medical care, home-based primary care, domiciliary support, community living centers, and behavioral health). Moreover, mental health problems frequently exist in addition to the physical health concerns veterans experience (Selim et al., 2004). Indeed, of the 4.5 million veterans screened in primary care settings, approximately a quarter, or 1.15 million, were diagnosed with at least one of five common mental health concerns (depression, posttraumatic stress disorder [PTSD], substance use disorder, anxiety, and serious mental illness; Trivedi et al., 2015). Psychologists play an increasingly important role in the VA as there is an ongoing effort to implement integrated mental health interventions (Zeiss \& Karlin, 2008). Thus, provision of services within the VA medical system that is sensitive to both medical and psychological concerns is vital to meeting the healthcare needs of veterans.

Veterans frequently view their military identity as distinct from their civilian self, and reintegration into society requires an adjustment and consolidation of these two identities (Orazem et al., 2017). Efforts to evaluate and understand veteran experience, and subsequently presentation of healthcare concerns, require consideration of an array of distinct service experiences (Armistead-Jehle, Soble, Cooper, \& 
Belanger, 2017; Coll \& Weiss, 2016). The era during which a veteran served in the military, and the associated country or region in which they were deployed, is a common descriptive characteristic for contextualizing an individual's military service history. For instance, compensation for injuries are categorized by periods of eligible service (Department of Veteran Affairs, 2018). Bumper stickers, descriptive apparel, self-affirming statements (e.g., "I am a Vietnam Vet"), and other expressive methods are often used to express the time and deployment locations occurring within their service record. Moreover, research frequently examines differences between service eras (e.g., Mclaughlin, Nielsen, \& Waller, 2008; Teigen, 2006; Wilmoth, London, \& Parker, 2010) or provides information on service era as a critical sample descriptive characteristic (e.g., Bellet, McDevittMurphy, Thomas, \& Luciano, 2018; Ingram, Tarescavage, Ben-Porath, \& Oehlert, 2019a, b; Mozley, Willer, Weathers, Beckham, \& Feldman, 2005).

Currently, the two largest groups of veterans receiving treatment within the VA healthcare system are those who served during the Vietnam era (roughly 1961-1975) and the Gulf War (1990 until present; Department of Veteran Affairs, 2018). These groups report a variety of substantially different wartime, homecoming, and medical experiences. They tend to not only seek treatment at different rates and in different ways (Boyd, Juanamarga, \& Hashemi, 2015; Rosenheck \& Fontana, 2008; Quatana et al., 2014), but also present with distinct sets of medical and diagnostic concerns stemming from actual or perceived wartime conditions (e.g., varying rates of exposure to blast concussive force, Gulf War Syndrome, and Agent Orange Exposure; Haley, Kurt, \& Hom, 1997; Stellman \& Stellman, 2018; Wells et al., 2011). Vietnam veterans report experiencing social rejection and feelings of betrayal lasting for over 30 years following their service (Desai, Pavlo, Harpaz-Rotem, \& Rosenheck, 2016). They are also more likely to apply for social security disability than Gulf War Veterans (Muller, Early, \& Ronca, 2014). Additionally, there are demographic differences between the groups, with Vietnam era veterans being more likely to have been married, less likely to be female, less likely to be working, and more likely to have a history of incarceration (Fontana \& Rosenheck, 2008; Maclean \& Elder, 2007).

In addition to those concerns outlined above, a number of studies have interpreted their findings in such a way as to suggest that psychiatric issues also vary across service era. Exemplifying this, Posttraumatic Stress Disorder (PTSD) is a common psychiatric issue for those with military service histories and impacts veterans in a manner that is disproportionate to the civilian population (Kilpatrick et al., 2013). Estimates suggest that between 13 and 14\% of veterans qualify for a PTSD diagnosis (e.g., Tanielian \& Jaycox, 2008 ) and an approximate $17 \%$ of the veterans receive disability compensation from the VA solely because of PTSD
(National Center for Veterans Analysis and Statistics, 2014). Due to both its impact and frequency within this population, PTSD is a public health epidemic for veterans (Dursa, Reinhard, Barth, \& Schneiderman, 2014). PTSD substantially impacts physical and mental well-being (Kyle, Wade, Andersen, \& Ouimette, 2010; Maguen et al., 2010), as well as social and family relationships (Galovski \& Lyons, 2004). Indeed, there is a substantive literature base documenting the relationship between negative health outcomes and PTSD. Chronic pain is a particularly common health concern which is associated with greater prevalence of PTSD (Fishbain, Pulikal, Lewis, \& Gao, 2017; Outcat, Hoen, Yu, Franks, \& Krebs, 2016), as a significant portion of those with chronic pain concerns report a history of trauma exposure (Åkerblom, Perrin, Fischer, \& McCracken, 2017). More broadly, those exposed to trauma, as well as those meeting diagnostic criteria for PTSD, are more likely to experience various physical health issues such as sleep disorders, respiratory problems, and migraines (El-Gabalawy, Blaney, Tsai, Sumner, \& Pietrzak, 2018). Moreover, health problems seen within those with PTSD include a broad array of issues relating to gastrointestinal, radiological, musculoskeletal, and general health concerns (Pacella, Hruska, \& Delahanty, 2013).

When contrasting among service eras, PTSD prevalence estimates have varied between 8 and 50\% across different wars and armed conflicts (e.g., Hoge \& Warner, 2014; McCarroll, Ursano, \& Fullerton, 1995; Stretch, Marlowe, Wright, Bliese, Knudson, \& Hoover, 1996; Wolfe, Brown, \& Kelley, 1993). Of those eras which predominate current VA service utilization (Department of Veteran Affairs, 2018), Vietnam era veterans have had notably higher prevalence rates than those from Gulf War deployments (Fulton et al., 2014; Kang, Natelson, Mahan, Lee, \& Murphy, 2003; Murphy, Johnson, Chung, \& Beaton, 2003; Weiss et al., 1994). Likewise, PTSD symptom severity has also been found to differ by service era, albeit with somewhat mixed results (e.g., Gros, Stachan, et al., 2011; Yoder et al., 2012). To some extent, discrepancies in symptom severity, differences in diagnostic patterns, prevalence across service eras may be a result of differences in the reliability and/or validity of the methods used to assess them (Brown, Grubaugh, Knapp, \& Acierno, 2016). In addition to symptom checklists and diagnostic interviews, which are subject to interviewer or evaluator bias, broadband measures with protocol validity scales are also used to evaluate PTSD. These are objective instruments that can be used to assess the array of clinical concerns central to PTSD (i.e., avoidance behaviors, intrusive symptoms, negative mood and cognitions, and alterations in arousal and reactivity; Sellbom, Lee, Ben-Porath, Arbisi, \& Gervais, 2012). They also evaluate common comorbid concerns which have important clinical implications (e.g., 
anger, aggression, depression, suicidality, hopelessness, substance use; Crowson, Frueh, \& Snyder, 2001; Jakupcak, Conybeare, Phelps, Hunt, \& Holmes, 2007; Trivedi et al., 2015). Importantly, broadband measures often have validity scales to evaluate the extent to which the test-taker provided an accurate report of their symptoms.

In a study by Glenn et al. (2002), the influence of service era was examined on one of the most commonly used broadband instruments, the Minnesota Multiphasic Personality Inventory-2 (MMPI-2; Butcher, Graham, BenPorath, Tellegen, \& Dahlstrom, 2001). After excluding approximately $45 \%$ of the initial sample because these individuals produced elevated MMPI-2 Validity Scale scores, Glenn et al. (2002) found that Gulf War veterans endorsed higher rates of hypomania, whereas Vietnam veterans reported greater rates of depression, anxiety, and social isolation. Those of different service eras also reported different rates of two-point code types, which are sometimes interpreted as representative of unique clinical presentations; Vietnam era veterans were more frequently classified as displaying 2-8/8-2 while Gulf veterans more frequently had a $1-8 / 8-1$ profile. In addition, they also found that health-related concerns were higher in Vietnam veterans, including those related to current health concerns as well as physician diagnosed health problems.

Notwithstanding the strengths of the Glenn et al. (2002) study (i.e., use of a broadband measure with validity scales), the psychometrics of MMPI-2 scale have long been called into question (e.g., Helmes \& Reddon, 1993). Some of these issues led to the development of the MMPI-2-Restructured Form (MMPI-2-RF; Tellegen \& Ben-Porath, 2008/2011), which also has validity scales and substantive scales with notably improved discriminant validity (Ben-Porath, 2012; Simms, Casillas, Clark, Watson, \& Doebbeling, 2005). To date, research on the MMPI-2-RF has established a notable base of literature supporting it as a useful diagnostic aide with veterans in a variety of healthcare settings within the VA. Exemplifying some of this literature, several core scales have demonstrated marked differences for those veterans with PTSD, including Demoralization (RCd), Somatic Concerns (RC1), Low Positive Emotions (RC2), and Dysfunctional Negative Emotions (RC7), all of which are consistently higher among veterans meeting diagnostic criteria (Wolf et al., 2008). In addition, the Anxiety (AXY) scale has been identified as a key indicator of PTSD symptomatology (e.g., Arbisi et al., 2011; Sellbom et al., 2012). Beyond use as an aid for assessing PTSD, the MMPI-2-RF substantive scales also predict a variety of clinically relevant criteria across other VA treatment settings, including substance use treatment programs (Forbey \& Ben-Porath, 2007), inpatient and outpatient mental health settings (Nelson, Anderson, Hoelzle, \& Arbisi, 2014), and primary care (Forbey, BenPorath, \& Arbisi, 2012; Ingram et al., 2019a).

\section{Present Study}

Service era represents a unique set of experiences, conditions, and perspectives which may impact the presentation and delivery of healthcare services to veterans across service setting. Past research demonstrates several differences across veterans from different service eras, including physical and mental health symptom severity, although research using well-validated, broadband measures with protocol validity scales is lacking. Research using the MMPI-2 has found distinct sets of presentation concerns for those evaluated for PTSD that necessitated specific evaluation and treatment needs for not only PTSD, but also related physical health conditions. However, no similar investigations have occurred using the MMPI-2-RF, despite research supporting the improved psychometrics of its scales as well as its utility in assessing veteran relevant health constructs. Therefore, this study utilizes the psychometrically sound MMPI-2-RF to examine differences in symptomatology across the Vietnam and Gulf War service eras in a national sample of VA outpatients.

\section{Method}

\section{Participants}

This study draws its sample from a large national study of VA patients assessed using either the MMPI-2 or MMPI2-RF between January 1st, 2008 and May 31st, 2015. This study included only those patients whose assessments were conducted in PTSD Clinical Team (PCT) settings. We excluded patients who scored above the recommended validity cut-scores on the MMPI-2-RF as such scores indicate invalid responding and bias substantive scale elevations (Ingram et al., 2019b). We excluded individuals whose electronic medical records indicated that they did not serve in Vietnam or Gulf War periods. These inclusion and exclusion efforts resulted in a valid sample of 1497 ( $n=792$ Gulf War and $n=510$ Vietnam). Table 1 provides demographic information for this study's sample. In general, participants are married, male, did not serve in combat, and mostly served during the Gulf War. Veterans from the Vietnam conflict were more likely to be married, male, and have $100 \%$ service connection. 
Table 1 Demographic information

\begin{tabular}{llll}
\hline & All & Era of service \\
\cline { 3 - 4 } & & Gulf War & Vietnam \\
\hline Male & $87.3 \%$ & $82.5 \%(n=792)$ & $95.9 \%(n=510)$ \\
& $(n=1,492)$ & & \\
Marital status & - & - & - \\
Single & $29.7 \%(n=443)$ & $27.4 \%(n=263)$ & $33.8 \%(n=180)$ \\
Married & $42.0 \%(n=626)$ & $36.8 \%(n=353)$ & $51.3 \%(n=273)$ \\
Divorced & $20.8 \%(n=311)$ & $28.2 \%(n=271)$ & $7.5 \%(n=40)$ \\
Widowed & $5.7 \%(n=85)$ & $6.7 \%(n=64)$ & $3.9 \%(n=21)$ \\
Other/missing & $0.6 \%(n=27)$ & $0.1 \%(n=9)$ & $3.4 \%(n=18)$ \\
Combat service & $17.5 \%(n=226)$ & $18.8 \%(n=152)$ & $15.3 \%(n=74)$ \\
$\%$ service con- & - & - & - \\
nected & & & \\
NSC or 0 & $1.3 \%(n=17)$ & $0.8 \%(n=7)$ & $2.1 \%(n=10)$ \\
10 & $4.0 \%(n=53)$ & $4.5 \%(n=38)$ & $3.2 \%(n=15)$ \\
20 & $1.7 \%(n=22)$ & $1.4 \%(n=12)$ & $2.1 \%(n=10)$ \\
30 & $4.9 \%(n=64)$ & $4.6 \%(n=38)$ & $4.3 \%(n=20)$ \\
40 & $5.2 \%(n=69)$ & $5.2 \%(n=44)$ & $4.7 \%(n=22)$ \\
50 & $6.1 \%(n=80)$ & $5.5 \%(n=47)$ & $7.0 \%(n=33)$ \\
60 & $8.9 \%(n=117)$ & $8.2 \%(n=70)$ & $10.0 \%(n=47)$ \\
70 & $18.4 \%(n=243)$ & $18.6 \%(n=158)$ & $18.1 \%(n=85)$ \\
80 & $16.8 \%(n=221)$ & $18.6 \%(n=158)$ & $13.4 \%(n=63)$ \\
90 & $10.8 \%(n=143)$ & $12.6 \%(n=107)$ & $7.7 \%(n=36)$ \\
100 & $22.0 \%(n=290)$ & $19.1 \%(n=162)$ & $27.3 \%(n=128)$ \\
\hline & & &
\end{tabular}

Frequencies were calculated out of those with available demographic information. Percentages were calculated based on available demographic information. Combat service status was available for 1290 (86.6\%) study participants and information about service connection was available on 1,319 participants $(88.4 \%)$. Age was not coded within the database utilized for analysis

\section{Instruments}

\section{MMPI-2-Restructured Form}

The MMPI-2-RF (Tellegen \& Ben-Porath, 2008/2011) is a 338 -item personality measure comprising 51 scales, which includes 9 validity indicators used to determine if a respondent's profile is interpretable. The validity scales of the MMPI-2-RF are effective at discerning feigned and actual symptoms but are sensitive to veteran status and veteran relevant issues (e.g., PTSD, neurological complaints; Ingram \& Ternes, 2016). The interpretive manual of the MMPI-2-RF specifies the following scale scores as indicating probable patterns of invalid responding: VRIN-r $\geq 80$, TRIN-r $\geq 80$, F- $r=120$, Fp- $r \geq 100$, Fs $\geq 100$, RBS $\geq 100$, FBS-r $\geq 100$, L-r $\geq 80$, and K-r $\geq 70$. Additionally, profiles may have compromised scale validity if an insufficient number of items are scorable (CNS $\geq 15)$. The remaining 42 substantive scales measure various clinical constructs and are structured hierarchically. These substantive scales include 3 Higher-Order construct scales, 9 clinical scales referred to as the Restructured Clinical (RC) scales, 23 specific problem scales, 2 interest scales, and the scales of the Personality Psychopathology 5 (PSY$5)$. Included within the 23 specific problem scales are 5 scales assessing explicit health-related concerns: Malaise (MLS), Gastrointestinal Complaints (GIC), Head Pain Complaints (HPC), Neurological Complaints (NUC), and Cognitive Complaints (COG). Scores on the substantive scales are measured using standardized uniform $T$ scores, with a score of 65 equating to approximately the $92 \mathrm{nd}$ percentile.

\section{Procedures}

This study utilized data which were extracted from the VA Informatics and Computing Infrastructure (VINCI). This platform allows IRB approved access to the Corporate Data Warehouse (CDW) where veteran medical records (including mental health assessments) have been electronically entered. From CDW, MMPI-2 and MMPI-2-RF item responses were extracted for all test administrations between January 1st, 2008 and May 31st, 2015. These item responses were used to calculate MMPI-2-RF scale scores, which is possible because all the $338 \mathrm{MMPI}-2-\mathrm{RF}$ items are included on the 567 item MMPI-2. Participant MMPI-2/RF responses included no item missing data. Two papers have been published using this database, one calculated context-specific comparison groups using the MMPI-2-RF substantive scale scores (Ingram et al., 2019a) and the second examined patterns of validity scale functioning (Ingram et al., 2019b). This paper expands existing analyses by exploring the impact of service era on clinical presentation.

For this investigation, we identified PCT using stop codes to provide comparable results to the previous study comparing service era on the MMPI-2 (i.e., Glenn et al., 2002). Stop codes are numeric values defining the type of clinic in which a clinical service is being conducted; this study included only those administrations which took part in a PTSD Clinical Team (Stop code 540). These specialty PTSD clinic treatment teams utilize evidence-based treatments and assessments for trauma-exposed individuals, most of whom meet diagnostic criteria for PTSD (e.g., 89-91\%; Glenn et al., 2002; Mozley et al., 2005). In this way, study participants were those identified by VA providers as warranting evaluation due to reported trauma exposure. Likewise, those identified as having trauma exposure are likely to experience substantive health-related concerns (El-Gabalawy et al., 2018). Because of the nature of service era coding within the VA electronic medical record system, the Gulf War period includes those individuals in Operation Enduring Freedom (OEF), Operation Iraqi Freedom (OIF), Operation New Dawn (OND), and the Gulf War. 


\section{Results}

A series of one-way ANCOVAs were planned to evaluate differences between Vietnam and Gulf War Veterans across the substantive scales of the MMPI-2-RF. Biological sex was entered as a covariate to account for gender differences in MMPI-2-RF scores, as $82.5 \%$ of Gulf War patients were male compared to $95.9 \%$ of Vietnam War veterans. Because the large sample size in this study led to a very high power to detect statistically significant effects, an a priori decision was made to only interpret clinically meaningful differences as defined by a medium effect size or the equivalent of 5 $T$-score points (Rosenthal, Rosnow, \& Rubin, 2000).

As presented in Tables 2 and 3, One-Way ANCOVAs indicated that all differences were of less than a moderate effect size as defined by Cohen $(d<|.50| ; 1988)$.
Additionally, all but six analyses were of a negligible effect size $(d<|.20|)$. In this subset of analyses, Gulf War Veterans scored slightly higher than Vietnam War Veterans on Gastrointestinal Complaints $(d=.25)$, Head Pain Complaints $(d=.31)$, Anxiety $(d=.24)$, and Disconstraint $(d=.21)$. In contrast, Vietnam War Veterans scored slightly higher than Gulf War Veterans on the Neurological Complaints $(d=.26)$ and Multiple Specific Fears Scales $(d=.37)$. Additionally, phi coefficients were calculated to compare rates of substantive scales elevation between service eras. In general, substantive scales shared similar T65 and T75 elevations with phi coefficients falling in the negligible range. Along with a pattern of small effect-size differences in the somatic scales, Vietnam War veterans had greater rates of T65 and T75 elevation than Gulf War veterans on Suicidal Ideation scale (SUI).

Table 2 ANCOVA results for service era with gender covaried for validity, higher-order, and restructured clinical scales

\begin{tabular}{|c|c|c|c|c|c|c|c|c|c|c|c|c|c|}
\hline \multirow[t]{2}{*}{ Scale } & \multicolumn{2}{|c|}{ Overall } & \multirow{2}{*}{$\begin{array}{l}\text { Gender (covariate) } \\
F(1,1489)\end{array}$} & \multicolumn{2}{|l|}{ Service era } & \multicolumn{3}{|c|}{ Gulf war $(n=960)$} & \multicolumn{3}{|c|}{ Vietnam $(n=532)$} & \multirow[t]{2}{*}{$\varphi \geq 65$} & \multirow[t]{2}{*}{$\varphi \geq 75$} \\
\hline & $M$ & SD & & $F(1,1489)$ & $d$ & $M$ & $\% \geq 65$ & $\% \geq 75$ & M & $\% \geq 65$ & $\% \geq 75$ & & \\
\hline Validity & - & - & - & - & - & - & - & - & - & - & - & - & - \\
\hline VRIN-r & 53.0 & 9.3 & 0.814 & 1.482 & .08 & 53.4 & - & - & 52.8 & - & - & - & - \\
\hline TRIN-r & 57.3 & 6.5 & 3.222 & 2.895 & -.11 & 56.7 & - & - & 57.3 & - & - & - & - \\
\hline F-r & 80.9 & 18.5 & 0.240 & 0.156 & -.03 & 80.5 & - & - & 80.9 & - & - & - & - \\
\hline Fp-r & 65.1 & 13.2 & 0.040 & $9.087 * *$ & .16 & 65.8 & - & - & 63.6 & - & - & - & - \\
\hline Fs & 70.9 & 12.7 & 0.163 & 0.340 & .04 & 71.1 & - & - & 71.1 & - & - & - & - \\
\hline FBS-r & 75.3 & 13.9 & $19.855 * * *$ & $6.108^{*}$ & .18 & 73.1 & - & - & 71.4 & - & - & - & - \\
\hline RBS & 71.2 & 15.5 & 0.243 & 2.257 & .09 & 76.0 & - & - & 74.8 & - & - & - & - \\
\hline L-r & 40.1 & 9.0 & 1.001 & 2.414 & .07 & 54.4 & - & - & 53.6 & - & - & - & - \\
\hline K-r & 54.4 & 9.5 & 0.033 & 0.041 & .01 & 40.1 & - & - & 40.0 & - & - & - & - \\
\hline Higher-order & - & - & - & - & - & - & - & - & - & - & - & - & - \\
\hline EID & 67.8 & 12.1 & $17.883 * * *$ & 0.797 & .00 & 69.1 & $64.5 \%$ & $34.4 \%$ & 69.7 & $62.4 \%$ & $32.9 \%$ & -0.02 & -0.01 \\
\hline THD & 59.4 & 12.4 & 0.283 & 3.148 & -.09 & 55.2 & $27.1 \%$ & $10.9 \%$ & 53.7 & $30.8 \%$ & $11.7 \%$ & 0.04 & 0.01 \\
\hline $\mathrm{BXD}$ & 57.1 & 11.3 & $55.456 * * *$ & $5.770 *$ & .05 & 59.2 & $28.9 \%$ & $9.2 \%$ & 60.4 & $26.3 \%$ & $6.2 \%$ & -0.03 & -0.05 \\
\hline Restructured clinical & - & - & - & - & - & - & - & - & - & - & - & - & - \\
\hline $\mathrm{RCd}$ & 66.4 & 11.5 & 12.093 & 1.801 & .11 & 68.6 & $63.2 \%$ & $33.8 \%$ & 67.8 & $57.7 \%$ & $28.8 \%$ & -0.05 & -0.05 \\
\hline $\mathrm{RC} 1$ & 69.1 & 11.7 & 4.560 & 0.152 & .00 & 69.7 & $69.0 \%$ & $30.7 \%$ & 69.9 & $67.7 \%$ & $28.4 \%$ & 0.02 & -0.02 \\
\hline $\mathrm{RC} 2$ & 68.6 & 14.2 & 4.456 & 0.255 & -.01 & 69.3 & $64.6 \%$ & $35.3 \%$ & 69.7 & $63.9 \%$ & $35.7 \%$ & -0.01 & $<.01$ \\
\hline $\mathrm{RC} 3$ & 58.6 & 11.4 & 0.104 & 0.166 & .02 & 58.6 & $35.2 \%$ & $6.5 \%$ & 58.3 & $34.6 \%$ & $4.5 \%$ & -0.01 & 0.03 \\
\hline $\mathrm{RC} 4$ & 59.2 & 11.4 & 7.637 & 0.287 & -.06 & 58.1 & $33.4 \%$ & $9.0 \%$ & 58.5 & $34.6 \%$ & $10.5 \%$ & 0.01 & 0.03 \\
\hline RC6 & 60.9 & 13.0 & 1.895 & 1.076 & .07 & 61.7 & $38.5 \%$ & $17.5 \%$ & 60.9 & $33.8 \%$ & $16.5 \%$ & -0.04 & -0.01 \\
\hline RC7 & 63.6 & 12.2 & $16.876^{* * *}$ & 0.578 & .08 & 65.3 & $52.1 \%$ & $22.2 \%$ & 64.8 & $46.1 \%$ & $21.6 \%$ & -0.06 & -0.01 \\
\hline RC8 & 61.7 & 11.6 & 1.760 & 0.609 & -.06 & 61.0 & $36.8 \%$ & $13.6 \%$ & 61.5 & $38.9 \%$ & $14.7 \%$ & 0.02 & 0.01 \\
\hline RC9 & 52.9 & 10.3 & $36.871 * * *$ & $13.244 * * *$ & .13 & 51.8 & $15.8 \%$ & $3.4 \%$ & 49.8 & $10.7 \%$ & $1.1 \%$ & $-0.07 *$ & $-0.07 *$ \\
\hline
\end{tabular}

No differences between service eras exceed Cohen's (1988) interpretive classifications for small, medium, or large effects. \% of elevations above T65 and T75 were not calculated for validity scales. Validity Scale means are following exclusion for invalid responding using standard exclusion criteria (VRIN-r $\geq 80$, TRIN-r $\geq 80, \mathrm{~F}-\mathrm{r} \geq 120$, Fp-r $\geq 100$ ). Means presented are averaged over gender

$* p<.05, * * p<.01, * * * p<.001 . F$ and $\varphi$ values without a $*$ indicate non-significance 
Table 3 ANCOVA results for service era with gender covaried for somatic/cognitive, internalizing, externalizing, interpersonal, and Psy-5 scales

\begin{tabular}{|c|c|c|c|c|c|c|c|c|c|c|c|c|c|}
\hline \multirow[t]{2}{*}{ Scale } & \multirow[t]{2}{*}{$M$} & \multirow[t]{2}{*}{ SD } & \multirow{2}{*}{$\begin{array}{l}\text { Gender (covariate) } \\
F(1,1489)\end{array}$} & \multicolumn{2}{|l|}{ Service era } & \multicolumn{3}{|c|}{ Gulf war $(n=960)$} & \multicolumn{3}{|c|}{ Vietnam $(n=532)$} & \multirow[t]{2}{*}{$\varphi \geq 65$} & \multirow[t]{2}{*}{$\varphi \geq 75$} \\
\hline & & & & $F(1,1489)$ & $d$ & $M$ & $\% \geq 65$ & $\% \geq 75$ & M & $\% \geq 65$ & $\% \geq 75$ & & \\
\hline Somatic/cognitive & - & - & - & - & - & - & - & - & - & - & - & - & - \\
\hline MLS & 72.1 & 11.2 & 3.141 & 3.291 & -.08 & 72.3 & $71.6 \%$ & $55.9 \%$ & 73.4 & $75.8 \%$ & $58.1 \%$ & 0.04 & 0.02 \\
\hline GIC & 64.2 & 15.7 & $14.961 * * *$ & $15.022 * * *$ & .25 & 67.1 & $46.7 \%$ & $29.7 \%$ & 63.8 & $35.2 \%$ & $19.9 \%$ & $-0.10 * * *$ & $-0.10 * * *$ \\
\hline HPC & 63.1 & 11.4 & $4.203^{*}$ & $26.943 * * *$ & .31 & 64.9 & $\mathbf{5 4 . 9 \%}$ & $21.0 \%$ & 61.7 & $38.9 \%$ & $10.7 \%$ & $-0.15 * * *$ & $-0.13 * * *$ \\
\hline NUC & 67.5 & 13.4 & 2.372 & $19.213 * * *$ & -.26 & 65.7 & $61.6 \%$ & $31.9 \%$ & 68.9 & $\mathbf{7 0 . 7 \%}$ & $42.3 \%$ & $0.09 * * *$ & $0.10 * * *$ \\
\hline $\mathrm{COG}$ & 70.0 & 13.4 & 0.247 & 10.420 & .18 & 71.1 & $65.1 \%$ & $52.1 \%$ & 68.7 & $56.8 \%$ & $43.8 \%$ & -0.08 & -0.08 \\
\hline Internalizing & - & - & - & - & - & - & - & - & - & - & - & - & - \\
\hline SUI & 58.0 & 17.0 & 0.604 & 31.528 & -.08 & 56.5 & $37.4 \%$ & $15.5 \%$ & 61.7 & $52.4 \%$ & $23.9 \%$ & $0.15^{* * *}$ & $0.10 * * *$ \\
\hline HLP & 59.6 & 13.8 & 0.377 & 7.129 & -.14 & 59.1 & $32.9 \%$ & $14.9 \%$ & 61.2 & $37.4 \%$ & $20.5 \%$ & 0.04 & $0.07 * *$ \\
\hline SFD & 60.3 & 12.3 & 28.327 & 0.412 & .09 & 62.4 & $49.6 \%$ & $30.8 \%$ & 62.0 & $46.2 \%$ & $25.2 \%$ & -0.03 & $-0.06^{*}$ \\
\hline NFC & 57.8 & 11.6 & 32.871 & 0.045 & .05 & 59.6 & $26.6 \%$ & $16.5 \%$ & 59.8 & $12.9 \%$ & $12.6 \%$ & -0.04 & -0.05 \\
\hline STW & 60.5 & 11.5 & 4.779 & 8.286 & .18 & 61.9 & $50.5 \%$ & $9.3 \%$ & 60.1 & $41.9 \%$ & $5.6 \%$ & $-0.08 * * *$ & $-0.06^{*}$ \\
\hline $\mathrm{AXY}$ & 76.7 & 15.5 & 0.366 & $17.692 * * *$ & .24 & 78.3 & $80.7 \%$ & $61.1 \%$ & 74.7 & $73.7 \%$ & $\mathbf{5 0 . 4 \%}$ & $-\mathbf{0 . 0 8} * * *$ & $-0.10 * * *$ \\
\hline ANP & 63.0 & 12.4 & 7.324 & 8.160 & .12 & 62.7 & $58.4 \%$ & $16.3 \%$ & 60.7 & $50.4 \%$ & $13.3 \%$ & $-0.08 * * *$ & -0.04 \\
\hline BRF & 57.7 & 12.8 & 37.086 & 0.027 & .07 & 60.0 & $23.1 \%$ & $10.2 \%$ & 59.9 & $18.4 \%$ & $7.3 \%$ & $-0.05^{* * *}$ & -0.05 \\
\hline MSF & 48.5 & 8.7 & 67.992 & $71.206 * * *$ & -.37 & 49.2 & $6.3 \%$ & $0.7 \%$ & 53.1 & $11.7 \%$ & $0.8 \%$ & 0.09 & $<.01$ \\
\hline Externalizing & - & - & - & - & - & - & - & - & - & - & - & - & - \\
\hline $\mathrm{JCP}$ & 55.7 & 12.5 & 12.435 & 2.372 & -.12 & 54.1 & $19.1 \%$ & $10.2 \%$ & 55.1 & $22.6 \%$ & $12.0 \%$ & 0.04 & 0.03 \\
\hline SUB & 56.7 & 12.9 & 4.682 & 3.001 & .07 & 56.4 & $25.9 \%$ & $14.0 \%$ & 55.1 & $21.8 \%$ & $11.1 \%$ & -0.04 & -0.04 \\
\hline AGG & 59.4 & 12.4 & 33.157 & 5.181 & .06 & 57.9 & $35.7 \%$ & $11.0 \%$ & 56.4 & $33.3 \%$ & $9.2 \%$ & -0.02 & -0.03 \\
\hline $\mathrm{ACT}$ & 51.1 & 11.3 & 6.263 & 0.099 & .04 & 52.0 & $18.3 \%$ & $8.0 \%$ & 51.8 & $11.8 \%$ & $4.7 \%$ & -0.08 & -0.06 \\
\hline Interpersonal & - & - & - & - & - & - & - & - & - & - & - & - & - \\
\hline FML & 55.8 & 11.6 & 35.327 & 0.066 & .08 & 57.9 & $20.6 \%$ & $5.6 \%$ & 57.7 & $20.3 \%$ & $4.9 \%$ & $<.01$ & -0.02 \\
\hline IPP & 47.6 & 9.8 & 43.414 & 8.319 & -.09 & 48.9 & $6.8 \%$ & $1.1 \%$ & 50.5 & $8.6 \%$ & $0.9 \%$ & 0.03 & $<-.01$ \\
\hline SAV & 65.7 & 12.6 & 2.060 & 0.133 & .00 & 65.2 & $63.0 \%$ & $40.1 \%$ & 65.0 & $64.7 \%$ & $41.2 \%$ & 0.02 & 0.01 \\
\hline SHY & 52.7 & 10.4 & 12.127 & 6.067 & -.10 & 53.2 & $19.2 \%$ & $7.4 \%$ & 54.7 & $22.6 \%$ & $11.8 \%$ & 0.04 & $0.07 * *$ \\
\hline DSF & 66.6 & 17.0 & 1.900 & 0.195 & -.04 & 65.8 & $55.1 \%$ & $36.8 \%$ & 66.2 & $55.6 \%$ & $38.0 \%$ & $<.01$ & 0.01 \\
\hline PSY-5 & - & - & - & - & - & - & - & - & - & - & - & - & - \\
\hline$A G G R$ & 56.0 & 11.9 & 83.814 & 12.733 & .10 & 53.7 & $31.8 \%$ & $7.5 \%$ & 51.4 & $26.7 \%$ & $3.4 \%$ & -0.05 & $-0.08 * *$ \\
\hline PSYC & 58.6 & 12.0 & 0.028 & 3.619 & -.10 & 58.2 & $26.3 \%$ & $10.4 \%$ & 59.4 & $31.0 \%$ & $10.2 \%$ & $0.05^{*}$ & $<-.01$ \\
\hline DISC & 56.0 & 10.4 & $94.393 * * *$ & $32.894 * * *$ & .21 & 54.2 & $24.2 \%$ & $7.9 \%$ & 51.1 & $17.9 \%$ & $4.1 \%$ & $-0.07 * *$ & $<-.02 * *$ \\
\hline NEGE & 64.8 & 12.5 & 7.588 & 0.073 & .04 & 65.7 & $52.7 \%$ & $25.1 \%$ & 65.7 & $51.9 \%$ & $25.8 \%$ & $<-.01$ & $<-.03$ \\
\hline INTR & 67.0 & 13.9 & 0.175 & 0.074 & -.02 & 66.8 & $57.5 \%$ & $31.3 \%$ & 67.0 & $57.3 \%$ & $33.6 \%$ & $<-.01$ & $<-.04$ \\
\hline
\end{tabular}

No differences between service eras exceed Cohen's (1988) interpretive classifications for medium or large effects for scale means. Small effect differences in scale means are bolded

${ }^{*} p<.05, * * p<.01, * * * p<.001 . F$ and $\varphi$ values without a $*$ indicate non-significance

\section{Discussion}

This study evaluated differences in mean scale scores and elevation frequencies on the substantive scales of the MMPI-2-RF across two distinct periods of military service in a nationally drawn sample of veterans assessed on a unit for PTSD treatment. Earlier research found service era differences for veterans evaluated on the MMPI- 2 that suggested different interpretive and treatment needs within the VA healthcare system (Glenn et al., 2002). Results from this study suggest four trends that are relevant for the use of, and research with, the MMPI-2-RF: (a) veterans assessed are generally homogeneous in their report of average symptom severity across service eras as significant scale differences are uncommon, (b) the magnitude of effect is small in instances where mean differences emerge, (c) patterns of symptom differences between eras on the MMPI-2-RF differ from the MMPI-2 and most differences reflect non-PTSD diagnostic concerns (i.e., somatic/cognitive complaints), and (d) service era differences are more identifiable when the frequency of clinical elevations $(\mathrm{T} \geq 65 / \mathrm{T} \geq 75$ ) is compared. 
Substantive scale means are generally similar across service eras; however, there are more differences in elevation frequencies which are statistically significant. These differences in elevation frequency are most observable on the Specific Problems (i.e., somatic/cognitive, internalizing, externalizing, and interpersonal) and PSY-5 Scales, rather than the Higher-Order and Restructured Clinical Scales. These differences were particularly pronounced for scales assessing constructs relevant to behavioral health settings, and those associated with trauma exposure. Vietnam veterans endorse Gastrointestinal Complaints (GIC) and Head Pain Complaints (HPC) less frequently; however, Neurological Complaints (NUC) are more common. A greater frequency of elevated NUC scores is consistent with an aging population that is more likely to experience neurological concerns, including problems with balance, motor coordination, and increased sensory problems. Those who served during the Gulf War had higher AXY and DISC-r, consistent with a more disinhibited and externalizing presentation. Such a presentation may partially reflect a developmental trajectory, especially for DISC-r, which captures elements of impulsivity that is higher among younger populations. Those scales also reflect a more severe exposure to trauma as many relate to core PTSD criteria (AXY; Sellbom, Lee, Ben-Porath, Arbisi, \& Gervais, 2012) and the course of symptom presentation (DISC-r; Sadeh, Miller, Wolf, \& Harkness, 2015).

Despite small differences in some health-related concerns and PTSD-related content, the magnitude of means and frequency of these scale elevations do not appear to differ enough to necessitate the use of separate comparison groups for these service eras in provision of healthcare services. While research documents a consistent relationship between PTSD and physical health concerns (Åkerblom et al., 2017; Pacella et al., 2013), substantial health-related differences do not emerge as a function of service era. Accordingly, this may be understood within the framework that the health issues common to those being evaluated for PTSD are a general response to disorder arousal symptoms (El-Gabalawy et al., 2018) and do not stem from specific service era or deployment experiences (e.g., rates of concussive force exposure, Agent Orange, or Gulf War Syndrome). This has direct clinical implications because the current MMPI-2-RF VA comparison groups do not include Gulf War veterans but can, nonetheless, be used with individuals of this service era. An ability to generalize findings across service eras is critical to improving assessment veracity, and subsequent treatment recommendations, as the MMPI-2-RF is widely used within the VA in a variety of healthcare services and clinic types (e.g., Ingram et al., 2019a; Russo, 2017).

Across both service eras, the rates at which both Vietnam and Gulf War veterans endorsed suicidal thinking are high. This elevation is consistent with the elevated risk of suicide in veterans (Kang, Bullman, Smolenski, Skopp,
Gahm, \& Reger, 2015), particularly those with trauma histories (Jakupcak, Cook, Imel, Fontana, Rosenheck, \& McFall, 2009). Alternatively, past research indicates that SUI scores are increased by the presence of potentially life-threatening medical problems (Tarescavage e al., 2013), which may explain why SUI elevations were more common among the older Vietnam veteran population. Nevertheless, clinicians should continue being mindful of the potential for suicidal risk - particularly within aging veterans. More generally, primary care settings appear to provide an optimal service location in which to regularly conduct those suicide screenings within the VA healthcare setting (Ashrafioun et al., 2016; Oslin et al., 2006) and the MMPI-2-RF has documented utility within various medical settings (Forbey et al., 2012; Ingram et al., 2019a), including for spinal surgery and bariatric evaluations (e.g., Block, Ben-Porath, \& Marek, 2013; Marek et al., 2012).

In contrast to the current investigation using the MMPI2-RF, past MMPI-2 research has yielded notable differences in symptomatology across service eras. As noted earlier, Glenn et al. (2002) examined MMPI-2 scores and found that Gulf War veterans endorsed higher rates of hypomania, whereas Vietnam veterans reported greater rates of depression, anxiety, and social isolation. Conversely, in this study there were no meaningful differences in these areas across service eras. These differences may reflect the improved discriminant validity of the MMPI2-RF scales. Additionally, whereas Glenn and colleagues' investigation of the MMPI-2 utilized a single over-reporting scale to exclude invalid protocols, we utilized all nine MMPI-2-RF Validity Scales. As such, substantive score comparisons in this study are much less influenced by response bias differences across service eras.

This study should be considered within the scope of its limitations. First, although a very high percentage of individuals in PCT teams meet criteria for PTSD (approximately 90\%; see Glenn et al., 2002; Mozley et al., 2005), the individuals assessed as part of this study were not screened for PTSD or other psychiatric diagnoses. Accordingly, it is possible that, to a small extent, scores comparisons vary across groups as a function of their diagnostic presentation. Additionally, veteran age, as well as additional deployment information (other than service era), was not available, which would have been beneficial in identifying service-related experiences and how they may mediate symptom presentation. Finally, only selfreport information was available for the current study. It would have been useful to further compare differences in symptom presentation on the MMPI-2-RF as well as clinician-rated structured interviews. Notwithstanding these limitations, this study's 7-year national sample provides a comprehensive evaluation of service-era differences and offers supplemental understanding about what 
considerations are important during assessments within the VA healthcare system.

\section{Compliance with Ethical Standards}

Conflict of interest Yossef Ben-Porath is a paid consultant to the MMPI Publisher, the University of Minnesota, and Distributor, Pearson. He receives research funds from the University of Minnesota and as co-author of the MMPI-2-RF, he receives royalties on sales of the test. Anthony Tarescavage receives research funding from the University of Minnesota Press. Paul Ingram receives research funding from Pearson Clinical Assessments. Mary E. Oehlert declares no conflicts of interest.

Human and Animal Rights All procedures performed in this study involving human participants were in accordance with the ethical standards of the institutional research committee and with the 1964 Helsinki declaration and its later amendments or comparable ethical standards. This article does not contain any studies with animals performed by any of the authors.

Informed Consent This study utilized an anonymous retrospective database on which analyses were conducted. As such, informed consent was obtained from all individual participants in this study for their inclusion.

\section{References}

Åkerblom, S., Perrin, S., Fischer, M. R., \& McCracken, L. M. (2017). The impact of PTSD on functioning in patients seeking treatment for chronic pain and validation of the posttraumatic diagnostic scale. International Journal of Behavior Medicine, 24, 249-259.

Arbisi, P. A., Polusny, M. A., Erbes, C. R., Thusras, P., \& Reddy, M. K. (2011). The Minnesota Multiphastic Personality Inventory-2 Restructured Form in National Guard soldiers screening positive for posttraumatic stress disorder and mild traumatic brain injury. Psychological Assessment, 23, 203-214. https://doi.org/10.1037/ a0021339.

Armistead-Jehle, P., Soble, J. R., Cooper, D. B., \& Belanger, H. G. (2017). Unique aspects of traumatic brain injury in military and veteran populations. Physical Medicine and Rehabilitation Clinics, 28, 323-337. https://doi.org/10.1016/j.pmr.2016.12.008.

Ashrafioun, L., Pigeon, W. R., Conner, K. R., Leong, S. H., \& Oslin, D. W. (2016). Prevalence and correlates of suicidal ideation and suicide attempts among veterans in primary care referred for a mental health evaluation. Journal of Affective Disorders, 189, 344-350.

Bellet, B. W., McDevitt-Murphy, M. E., Thomas, D. H., \& Luciano, M. T. (2018). The utility of the personality assessment inventory in the assessment of posttraumatic stress disorder in $\mathrm{OEF} /$ OIF/OND veterans. Assessment, 25, 1074-1083. https://doi. org/10.1177/1073191116681627.

Ben-Porath, Y. S. (2012). Interpreting the MMPI-2-RF. Minneapolis: University of Minnesota Press.

Block, A. R., Ben-Porath, Y. S., \& Marek, R. J. (2013). Psychological risk factors for poor outcome of spinal surgery and spinal cord simulator implant: A review of the literature and their assessment with the MMPI-2-RF. The Clinical Neuropsychologist, 27, 81-107. https://doi.org/10.1080/13854046.2012.721007.

Boyd, J. E., Juanamarga, J., \& Hashemi, P. (2015). Stigma of taking psychiatric medications among psychiatric outpatient veterans.
Psychiatric Rehabilitation Journal, 38, 132-134. https://doi. org/10.1037/prj0000122.

Brown, W., Grubaugh, A. L., Knapp, R. G., \& Acierno, R. E. (2016). Interplay between service era, PTSD symptom expression, and treatment completion among veterans. Military Psychology, 28, 418-428. https://doi.org/10.1037/mil0000133.

Butcher, J. N., Graham, J. R., Ben-Porath, Y. S., Tellegen, A., \& Dahlstrom, G. (2001). Minnesota Multiphasic Personality Inventory-2: Manual for administration, scoring, and interpretation. Minneapolis, MN: University of Minnesota Press.

Coll, J. E., \& Weiss, E. L. (2016). Caring for the Military: A guide for helping professionals. New York: Routledge.

Crowson, J. J., Frueh, B. C., \& Snyder, C. R. (2001). Hostility and hope in combat-related posttraumatic stress disorder: A look back at combat compared to today. Cognitive Therapy and Research, $25,149-165$.

Department of Veteran Affairs. (2018). National Center for Veterans Analysis and Statistics. Retrieved from https://www.va.gov/vetda ta/Veteran_Population.asp

Desai, M. U., Pavlo, A. J., Harpaz-Rotem, I., \& Rosenheck, R. (2016). "I want to come home": Vietnam-era veterans' presenting for mental health care, roughly 40 years after Vietnam. Psychiatric Quarterly, 87, 229-239. https://doi.org/10.1007/s11126-015-9382-2.

Dursa, E. K., Reinhard, M. J., Barth, S. K., \& Schneiderman, A. L. (2014). Prevalence of a positive screen for PTSD among OEF/ OIF and OEF/OIF-era veterans in a large population-based cohort. Journal of Traumatic Stress, 27, 542-549. https://doi.org/10.1002/ jts. 21956.

El-Gabalawy, E., Blaney, C., Tsai, J., Sumner, J. A., \& Pietrzak, R. H. (2018). Physical health conditions associated with full and subthreshold PTSD in U.S. military veterans: Results from the National Health and Resilience in Veterans Study. Journal of Affective Disorders, 227, 849-853. https://doi.org/10.1016/j. jad.2017.11.058.

Fishbain, D. A., Pulikal, A., Lewis, J. E., \& Gao, J. (2017). Chronic pain types differ in their reported prevalence of post -traumatic stress disorder (PTSD) and there is consistent evidence that chronic pain is associated with PTSD: An evidence-based structured systematic review. Pain Medicine, 18, 711-735. https://doi. org $/ 10.1093 / \mathrm{pm} / \mathrm{pnw} 065$.

Fontana, A., \& Rosenheck, R. (2008). Treatment-seeking veterans of Iraq and Afghanistan: Comparison with veterans of previous wars. Journal of Nervous and Mental Disease, 196, 513-521. https:// doi.org/10.1097/NMD.0b013e31817cf6e6.

Forbey, J. D., \& Ben Porath, Y. S. (2007). A comparison of the MMPI-2 restructured clinical (RC) scales in a substance abuse treatment sample. Psychological Services, 4, 46-58.

Forbey, J. D., Ben Porath, Y. S., \& Arbisi, P. A. (2012). The MMPI-2 Computer Adaptive Version (MMPI-2 CA) in a medical outpatient facility. Psychological Assessment, 24, 628-639.

Fulton, J. J., Calhoun, P. S., Wagner, H. R., Schry, A. R., Hair, L. P., Elbogen, E., \& Beckham, J. C. (2014). The prevalence of posttraumatic stress disorder in Operation Enduring Freedom/Operation Iraqi Freedom (OEF/OIF) Veterans: A meta-analysis. Journal of Anxiety Disorders, 31, 98-107. https://doi.org/10.1016/j.janxd is. 2015.02 .003 .

Galovski, T., \& Lyons, J. A. (2004). Psychological sequelae of combat violence: A review of the impact of PTSD on veteran's family and possible interventions. Aggression and Violent Behavior, 9, 477-501. https://doi.org/10.1016/S1359-1789(03)00045-4.

Glenn, D. M., Beckham, J. C., Sampson, W. S., Feldman, M. E., Hertzberg, M. A., \& Moore, S. D. (2002). MMPI-2 profiles of Gulf and Vietnam combat veterans with chronic posttraumatic stress disorder. Journal of Clinical Psychology, 58, 371-381.

Gros, D. F., Stachang, M., Ruggiero, K. J., Knapp, R. B., Frueh, B. C., Egede, L. E., ... Acierno, R. (2011). Innovative service delivery 
for secondary prevention of PTSD in at-risk OIF-OEF service men and women. Contemporary Clinical Trials, 32, 122-128. https://doi.org/10.1016/j.cct.2010.10.003.

Haley, R. W., Kurt, T. L., \& Hom, J. (1997). Is there a Gulf War Syndrome? Searching for syndromes by factor analysis of symptoms. Journal of the American Medical Association, 277, 215-222. https ://doi.org/10.1001/jama.1997.03540270041025.

Helmes, E., \& Reddon, J. R. (1993). A perspective on developments in assessing psychopathology: A critical review of the MMPI and MMPI-2. Psychological Bulletin, 113(3), 453-471. https://doi. org/10.1037/1040-3590.17.3.345.

Hoge, C. W., \& Warner, C. H. (2014). Estimating PTSD prevalence in US veterans: Considering combat exposure, PTSD checklist cutpoints, and DSM-5. Journal of Clinical Psychiatry, 75, 14391441. https://doi.org/10.4088/JCP.14com09616.

Ingram, P.B., Tarescavage, A.M., Ben-Porath, Y.S., \& Oehlert, M.E. (2019a). Descriptive profiles of the MMPI-2-Restructured Form (MMPI-2-RF) across four Veteran Affairs treatment settings. Journal of Psychopathology and Behavioral Assessment. https:// doi.org/10.1007/s10862-019-09727-0

Ingram, P.B., Tarescavage, A.M., Ben-Porath, Y.S., \& Oehlert, M.E. (2019b). The MMPI-2-Restructured Form (MMPI-2-RF) validity scales: Patterns observed across veteran affairs settings [Measurement-Based Care and Psychological Assessment in Mental Health Services]. Psychological Services. https://doi.org/10.1037/ser00 00339

Ingram, P. B., \& Ternes, M. (2016). The detection of content-based invalid responding: A meta-analysis of the MMPI-2-Restructured Form's (MMPI-2-RF) over-reporting scales. The Clinical Neuropsychologist, 30, 473-496. https://doi.org/10.1080/13854 046.2016.1187769.

Jakupcak, M., Conybeare, D., Phelps, L., Hunt, S., \& Holmes, H. A. (2007). Anger, hostility, and aggression among Iraq and Afghanistan War veterans reporting PTSD and subthreshold PTSD. Journal of Traumatic Stress, 20, 945-954. https://doi.org/10.1002/ jts. 20258

Jakupcak, M., Cook, J., Imel, Z., Fontana, A., Rosenheck, R., \& McFall, M. (2009). Posttraumatic stress disorder as a risk factor for suicidal ideationinIraq and Afghanistan War Veterans. Journal of Traumatic Stress, 22, 303-306. https://doi.org/10.1002/ jts.20423.

Kang, H. K., Bullman, T. A., Smolenski, D. J., Skopp, N. A., Gahm, G. A., \& Reger, M. A. (2015). Suicide risk among 1.3 million veterans who were on active duty during the Iraq and Afghanistan wars. Annals of Epidemiology, 25, 96-100. https://doi. org/10.1016/j.annepidem.2014.11.020.

Kang, H. K., Natelson, B. H., Mahan, C.M., Lee, K. Y., \& Murphy, F. M. (2003). Post-traumatic stress disorder and chronic fatigue illnesses among Gulf War veterans: A population-based survey of 30,000 veterans. American Journal of Epidemiology, 157, 141-148. https://doi.org/10.1093/aje/kwf187.

Kilpatrick, D. G., Resnick, H. S., Milanak, M. E., Miller, M. W., Keyes, K. M., \& Friedman, M. J. (2013). National estimates of exposure to traumatic events and PTSD prevalence using DSM-IV and DSM-5 criteria. Journal of Traumatic Stress, 26, 537-547.

Kyle, P., Wade, M., Andersen, J., \& Ouimette, P. (2010). The impact of PTSD, depression, and substance use disorders on disease burden and health care utilization among OEF/OIF veterans. Psychological Trauma: Theory, Research, Practice, and Policy, 2, 218-223. https://doi.org/10.1037/a0019236.

Maclean, A., \& Elder, G. H. (2007). Military service in the life course. Annual Review of Sociology, 33, 175-196. https://doi. org/10.1146/annurev.soc.33.040406.131710.

Maguen, S., Lucenko, B. A., Reger, M. A., Gahm, G. A., Litz, B. T., ... \& Seal, K. H. (2010). The impact of reported direct and indirect killing on mental health symptoms in Iraq war veterans. The
Journal of Traumatic Stress, 23, 86-90. https://doi.org/10.1002/ jts.20434.

Marek, R.J., Ben-Porath, Y.S., Windover, A., Tarescavage, A.M., Merrell, J., Ashton, K., ... Heinberg, L.J. (2012). Assessing psychosocial functioning of bariatric surgery candidates with the Minnesota Multiphasic Personality Inventory-2 Restructured Form (MMPI-2-RF). Obesity Surgery, 23, 1864-1873.

McCarroll, J. E., Ursano, R. J., \& Fullerton, C. S. (1995). Symptoms of PTSD following recovery of war dead: 13-15-month follow-up. American Journal of Psychiatry, 152, 939-941.

McLaughlin, R., Nielsen, L., \& Waller, M. (2008). An evaluation of the effect of military service on mortality: Quantifying the healthy soldier effect. Annals of Epidemiology, 18, 928-936. https://doi. org/10.1016/j.annepidem.2008.09.002.

Mozley, S. L., Willer, M. W., Weathers, F. W., Beckham, J. C., \& Feldman, M. E. (2005). Personality Assessment Inventory (PAI) profiles of male veterans with combat-related posttraumatic stress disorder. Journal of Psychopathology and Behavioral Assessment, 27, 179-189. https://doi.org/10.1007/s10862-005-0634-6.

Muller, L. S., Early, N., \& Ronca, J. (2014). Veterans who apply for social security disabled-worker benefits after receiving a department of veterans affairs rating of "total disability" for serviceconnected impairments: Characteristics and outcomes. Social Security Bulletin, 74(3), 1-38.

Murphy, S. A., Johnson, L. C., Chung, I., \& Beaton, R. D. (2003). The prevalence of PTSD following the violent death of a child and predictors of change 5 years later. Journal of Traumatic Stress, $16,17-25$.

National Center for Veterans Analysis and Statistics. (2014). Trends in the Utilization of VA Programs and Services. Retrieved from https://www.va.gov/vetdata/docs/QuickFacts/Utilization_trend s_2014.PDF

Nelson, N. W., Anderson, C. R., Hoelzle, J. B., \& Arbisi, P. A. (2014). Psychological assessment of veterans in outpatient mental health settings. In S. S. Bush (Ed.), Psychological assessment of veterans (pp. 17-50). New York: Oxford University Press.

Orazem, R. J., Frazier, P. A., Schnurr, P. P., Oleson, H. E., Carlson, K. F., Litz, B. T., \& Sayer, N. A. (2017). Identity adjustment among Afghanistan and Iraq war veterans with reintegration difficulty. Psychological Trauma: Theory, Research, Practice, and Policy, 9(Suppl 1), 4-11. https://doi.org/10.1037/tra0000225.

Oslin, D. W., Ross, J., Sayers, S., Murphy, J., Kane, V., \& Katz, I. R. (2006). Screening, assessment, and management of depression in VA primary care clinics: The Behavioral Health Laboratory. Journal of General Internal Medicine, 21, 46-50.

Outcat, S. D., Hoen, H. M., Yu, Z., Franks, T. M., \& Krebs, E. E. (2016). Does comorbid chronic pain affect posttraumatic stress disorder diagnosis and treatment? Outcomes of posttraumatic stress disorder screening in Department of Veterans Affairs primary care. Journal of Rehabilitation Research and Development, 53, 37-44. https://doi.org/10.1682/JRRD.2014.10.0237.

Pacella, M. L., Hruska, B., \& Delahanty, D. L. (2013). The physical health consequences of PTSD and PTSD symptoms: A metaanalytic review. Journal of Anxiety Disorders, 27, 33-46. https:// doi.org/10.1016/j.janxdis.2012.08.004.

Quatana, P. J., Wilk, J. E., Thomas, J. L., Bray, R. M., Olmsted, K. L. R., Brown, J. M., \& Williams, J. (2014). Trends in mental health service utilization and stigma in US soldiers from 2002 to 2014. American Journal of Public Health, 104, 1671-1679. https://doi. org/10.2105/AJPH.2014.301971.

Rosenheck, R. A., \& Fontana, A. F. (2008). Recent trends in VA treatment of post-traumatic stress disorder and other mental disorders. Health Affairs. https://doi.org/10.1377/hlthaff.26.6.1720.

Rosenthal, R., Rosnow, R. L., \& Rubin, D. B. (2000). Contrasts and effect sizes in behavioral research: A correlational approach. New York: Cambridge University Press. 
Russo, A. C. (2017). A practitioner survey of department of Veteran Affairs Psychologists who provide neuropsychological assessments. Archives of Clinical Neuropsychology, 33, 1046-2059. https://doi.org/10.1093/arclin/arx139.

Sadeh, N., Harkness, M. W., \& Harkness, K. L. (2015). Negative emotionality and disconstraint influence PTSD symptom course via exposure to new major adverse life events. Journal of Anxiety Disorders, 31, 20-27. https://doi.org/10.1016/j.janxdis.2015.01.003.

Selim, A. J., Fincke, B. G., Ren, X. S., Lee, A., Rogers, W. H., Miller, D. R.,... \& Kazis, L. E. (2004). Comorbidity assessments based on patient report: Results from the Veterans Health Study. The Journal of ambulatory care management, 27, 281-295.

Sellbom, M., Lee, T. T. C., Ben-Porath, Y. S., Arbisi, P. A., \& Gervais, R. O. (2012). Differentiating PTSD symptomatology with the MMPI-2-RF (Restructured Form) in a forensic disability sample. Psychiatry Research, 197, 172-179.

Simms, L. J., Casillas, A., Clark, L. A., Watson, D., \& Doebbeling, B. N. (2005). Psychometric evaluation of the restructured clinical scales of the MMPI-2. Psychological Assessment, 17, 345-358.

Stellman, J. M., \& Stellman, S. D. (2018). Agent orange during the Vietnam war: The lingering issues of its civilian and military health impact. American Journal of Public Health, 108, 726-728. https://doi.org/10.2105/AJPH.2018.304426.

Stretch, R. H., Marlowe, D. H., Wright, K. M., Bliese, P. D., Knudson, K. H., \& Hoover, C. H. (1996). Posttraumatic stress disorder symptoms among Gulf War veterans. Military Medicine, 161, 407-410.

Tanielian, T., \& Jaycox, L. H. (Eds.). (2008). Invisible wounds of war: Psychological and cognitive injuries, their consequences, and services to assist recovery. Santa Monica, CA: RAND MG-720.

Tarescavage, A., Windover, A., Ben-Porath, Y., Boutacoff, L., Marek, R., Ashton, K., \& Heinberg, L. (2013). Use of the MMPI-2-RF suicidal/death ideation and substance abuse scales in screening bariatric surgery candidates. Psychological Assessment, 25, $1384-1389$.

Teigen, J. M. (2006). Enduring effects of the uniform: Previous military experience and voting turnout. Political Research, 59, 601-607. https://doi.org/10.1177/106591290605900409.

Tellegen, A., \& Ben-Porath, Y. S. (2008/2011). The Minnesota Multiphasic Personality Inventory-2 restructured form: Technical manual. Minneapolis, MN: University of Minnesota Press.

Trivedi, R. B., Post, E. P., Sun, H., Pomerantz, A., Saxon, A. J., Piette, J. D.,... \& Nelson, K. (2015). Prevalence, comorbidity, and prognosis of mental health among US veterans. American journal of public health, 105, 2564-2569.
United States Census Bureau. (2017). Profile America Facts for Figures: Veterans Day 2017 Nov. 11 (Report No. CB17-FF.22). Retrieved from https://www.census.gov/newsroom/facts-for-featu res/2017/veterans-day.html

Veterans Benefit Administration. (2017). Annual benefits report: Fiscal year 2017. Washington, DC: Department of Veterans Affairs.

Weiss, D. S., Marmar, C. R., Schlenger, W. E., Fairbank, J. A., Jordan, B. K., Hough, R. L., \& Kulka, R. A. (1994). The prevalence of lifetime and partial post-traumatic stress disorder in Vietnam theater veterans. Journal of Traumatic Stress, 5, 365-376. https ://doi.org/10.1002/jts.2490050304.

Wells, T. S., Miller, S. C., Adler, A. B., Engel, C. C., Smith, T. C., \& Fairbank, J. A. (2011). Mental health impact of the Iraq and Afghanistan conflicts: A review of US research, service provision, and programmatic responses. International Review of Psychiatry, 23, 144-152. https://doi.org/10.3109/09540261.2011.558833.

Wilmoth, J. M., London, A. S., \& Parker, W. M. (2010). Military service and men's health trajectories in later life. Journal of Gerontology: Social Sciences, 65B, 755-755. https://doi.org/10.1093/ geronb/gbq072.

Wolf, E. J., Miller, M. W., Orazem, R. J., Weierich, M. R., Castillo, D. T., Milford, J., ... Keane, T. M. (2008). The MMPI-2 restructured clinical scales in the assessment of posttraumatic stress disorder and comorbid disorders. Psychological Assessment, 20, 327-340. https://doi.org/10.1037/a0012948.

Wolfe, J., Brown, P. J., \& Kelley, J. M. (1993). Reassessing war stress: Exposure and the Gulf War. Journal of Social Issues, 49, 15-31.

Yoder, M., Tuerk, P. W., Price, M., Grubaugh, A. L., Strachan, M., Myrick, H., \& Acierno, R. (2012). Prolonged exposure therapy for combat-related posttraumatic stress disorder: Comparing outcomes for veterans of different wars. Psychological Services, 9, 16-25. https://doi.org/10.1037/a0026279.

Zeiss, A. M., \& Karlin, B. E. (2008). Integrating mental health and primary care services in the Department of Veteran Affairs Health Care System. Journal of Clinical Psychology in Medical Settings, 15, 73-78.

Publisher's Note Springer Nature remains neutral with regard to jurisdictional claims in published maps and institutional affiliations. 